\title{
Information models for problems solving of hardware design of multi-range chemical industries
}

\author{
Vladimir Mokrozub ${ }^{1, *}$, Evgeniy Malygin ${ }^{1}$, and Vladimir Nemtinov ${ }^{1}$ \\ ${ }^{1}$ Tambov State Technical University, 392000 Tambov, Sovetskaya 106, Russia
}

\begin{abstract}
The information models for determining sizes of apparatuses in chemical-technological systems and the development of separate technological apparatus are provided. The information model for solving the problem of calculating the determining sizes of apparatuses is based on the representation the structure of the chemical-technological system in the form of oriented polychromatic hypergraph, the edges of which are the products, and the vertices are the stages of the technological process. The information model for solving the problem of developing a separate technological apparatus allows determining its structure, the parameters of its elements and their location in the 3D model of the assembly. The structure of the apparatus is presented as a hierarchical system of hypergraphs, which was built on the base of the «and - or» tree of possible elements of the apparatus and the rules, that allow to select the necessary elements of «and - or» tree, depending on the functions of the apparatus and the conditions of its operation. The location of the elements of apparatus in space is determined by logical conditions describing their position relative to each other in the 3D model of the assembly.
\end{abstract}

\section{Introduction}

The development of processing industries, including the chemical industry, is one of the main tasks of the Russian economics. In accordance with the strategy for the development of the chemical and petrochemical industry in Russia for the period up to 2030 the Russian Federation provides for the modernization of working and development of new chemical industries, including multi-range chemical industry (MCI) such as the production of varnishes, paints, various additives, etc. These industries largely determine the quality of products of other industries: textile, automotive, rubber, technical, and other.

Formation of new and modernization of existing MCI is impossible without the development of effective hardware design of chemical-technological systems (CTS), consisting, as a rule, of a large number of technological apparatus (TA). The decisionmaking system in this case is a multi-level hierarchical system of interrelated local tasks, which should be provided with information of various types, means of storing and

\footnotetext{
*Corresponding author: mokr@mail.gaps.tstu.ru
} 
processing the results of solving local problems, the formation of design documentation necessary for the manufacture of TA with minimal human participation.

Information support decision-making system (ISDS) should be created for performing a number of functions. This system ensures the acceleration of decision-making processes, eliminating errors in the information transfer between local tasks of making design decisions, formalizing knowledge of the subject, processing and storing knowledge, reducing the number of design and technological services of enterprises.

Existing ISDS systems [1-4] in CTS design have fundamental shortcomings, the main one of which is the lack of decision making with the use of knowledge of the subject area, which in practice is compensated by the increase in the number of engineering departments of enterprises.

The task of CTS hardware design is decomposed into two interrelated subtasks $[5,6]$ :

- calculation of the number and determining sizes of CTS apparatus;

- development of individual apparatus design.

The basis of ISDS system for solving these problems are information models (IM), which represent a formalized set of facts, concepts, instructions intended for meeting the information needs of the solved tasks.

Information models and the results of solving specified tasks should be stored and processed in the selected information store, which is most often used as relational database.

The formalized representation of the structure of technical developed facilities makes it possible to significantly reduce the costs associated with the storage and processing of information. It is suggested to use graphs as a formal representation.

\section{The problem of calculation the number and determining dimensions of CTS apparatus}

In works $[5,6]$ the problem of determining the number and sizes of apparatuses at each stage of the technological system is set and solved in the following form. For given initial data:

- product range $P=\left\{p_{i}\right\}, i=\overline{1, I}, i$ - number of product;

- output of each product $Q=\left\{q_{i}\right\}, i=\overline{1, I}$;

- period of all products manufacturing $T$;

- list of stages (main CTS apparatus) $Y=\left\{y_{j}\right\}, j=\overline{1, J}, j$ - number of stage;

- list of stages (apparatus) used to produce each product $Y P=\left\{y p_{i k}\right\}, i=\overline{1, I}, k \in \overline{1, J}, Y P \subseteq Y$;

- operating time of each apparatus for one group of product manufacturing $T A=\left\{t a_{i j}\right\}, i=\overline{1, I}, j=\overline{1, J}$.

Need to find:

- determining geometrical dimensions (working volumes, surfaces) $X=\left\{x_{j}\right\}, j=\overline{1, J}$ of main apparatus of each stage. At that $X \subset X 1=\left\{x 1_{j}\right\}, j=\overline{1, J}$, where $X 1$ set of acceptable values of basic sizes of apparatus (catalog of apparatus);

- number of main apparatuses of each stage $N=\left\{n_{j}\right\}, j=\overline{1, J}$;

- batch sizes of finished product $W=\left\{w_{i}\right\}, i=\overline{1, I}$;

- start and end of each batch of product processing at each stage $T N_{i j}=\left\{t n_{k}\right\}, T K_{i j}=\left\{t k_{k}\right\}, i=\overline{1, I}, j=\overline{1, J}, r=1, K_{i}, k$ - batch number of $i$-product. 
The task is set as optimization task, the optimization criterion is the capital and operating costs of $S 1$, i.e.:

$$
\begin{aligned}
& I_{11}^{*}=\arg \min \left(S 1\left(I_{11}\right)\right), \\
& I_{11}=<X, N, W, T N, T K>=M_{11}(t z), \\
& t z=<P, Q, T, Y, Y P, T A>
\end{aligned}
$$

where $M_{11}$ - mathematical model that allows a set of initial data $t z$ find many results $I_{11}$.

Thus, the information model $I M_{11}$ can be represented in the form

$$
I M_{11}=<M_{11}, X 1, S 1>
$$

As shown above, the CTS consists of separate, interconnected apparatuses where operations are performed according to the technological regulations. When solving the problem of the number and size of devices choice we know the list of products; the stages required for the release of each product; type of apparatus at each stage of CTS.

The structure of CTS can be represented in the form of oriented hypergraph $G_{g o}(X, U)$, where $X=\left\{x_{i}\right\}, i=\overline{1, I}-$ set of vertices of hypergraph (process stages), $x_{i}$ - $i$ vertices; $U=\left\{u_{m}\left(Y_{m}\right)\right\}, \quad m=\overline{1, M}$ - set of edges of hypergraph (manufactured products), $u_{m}\left(Y_{m}\right)-m$ - edge of hypergraph, $Y_{m}$ - set of vertices incident to $m$-edge $Y_{m} \subseteq X, Y_{m}=\left\{x_{k}^{l}\right\}, k \in K_{m}, K_{m} \in \overline{1, I}, l^{l}$ - number of vertices in the edge of oriented hypergraph $l=\overline{1, L_{m}}$.

Figure 1 shows an example of representation in the form of a polychromatic oriented hypergraph $G_{g o}(X, U)$ CTS structures, designed for the manufacturing of two products $U=\left\{u_{1}\left(x_{1}, x_{2}, x_{4}, x_{3}\right), u_{2}\left(x_{1}, x_{2}, x_{4}, x_{5}\right)\right\}$.

For $u_{1}$ product manufacturing the stages (vertices) $x_{1}, x_{2}, x_{3}, x_{4}$ are used in the following sequence $x_{1}, x_{2}, x_{4}, x_{3}$. For $u_{2}$ product manufacturing the stages (vertices) $x_{2}, x_{3}, x_{4}, x_{5}$ are used in the following sequence $x_{2}, x_{4}, x_{3}, x_{5}, X=\left\{x_{1}, x_{2}, x_{3}, x_{4}, x_{5}\right\}-$ set of stages.

The edges (products) have the following properties (colors):

a) product name; b) volume of the product; c) sizes of finished product batches. The values of properties a) and b) are given, value of property c) must be found.

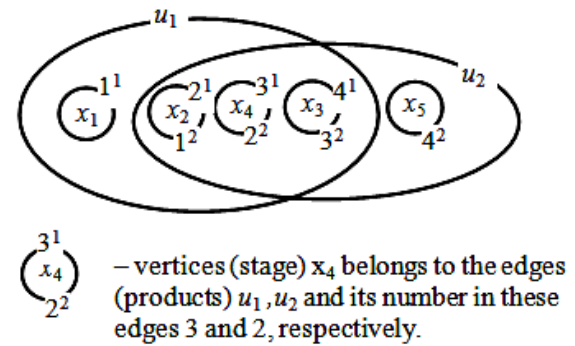

Fig. 1. Example of oriented polychromatic hypergraph technological scheme for two products manufacturing.

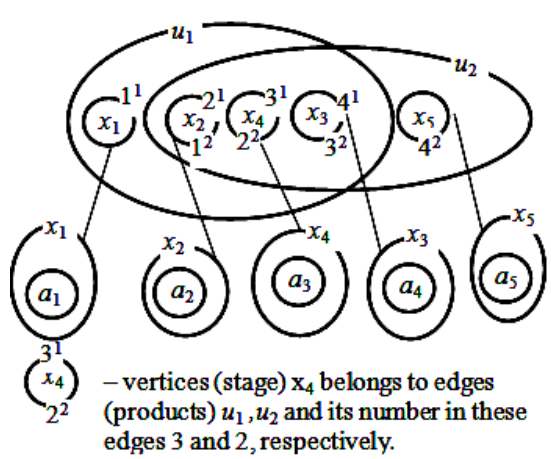

Fig. 2. Initial state of graphs for solving the problem of calculating the number and size of apparatuses 
Vertices (stages) have the following properties: a) name; b) type and name of apparatus; c) determining the geometric dimensions (working volumes, surfaces) of apparatus; d) number of devices; e) start and end for each batch of product. The values of properties a) and b) are given, the value of properties c), d), e) must be found.

Consequently, the problem of calculating the number and size of devices for the products output at each stage consists in finding the values of certain properties of the edges and vertices, namely: the sizes of batches of finished product, determining the geometric sizes of the apparatus (working volumes, surfaces); number of devices; the moments of start and end of the processing of each batch of product.

The presentation of CTS in the form of a hypergraph, where the edges are products, and the vertices - stages has the following limitations:

- in case of using several apparatus at the stage their defining geometric sizes should be the same;

- problem solution does not mean the addition of new apparatuses into the process chain, for example, transitional tanks.

You can neglect these restrictions if:

- consider as the vertices of hypergraph the apparatuses but not stages;

- stage becomes not a top, but an edge which unites several apparatuses;

- when solving a problem, new vertices (apparatuses), for example, intermediate capacitors, can be added.

- after or before a certain stage several new devices can be introduced, which are united by a new edge.

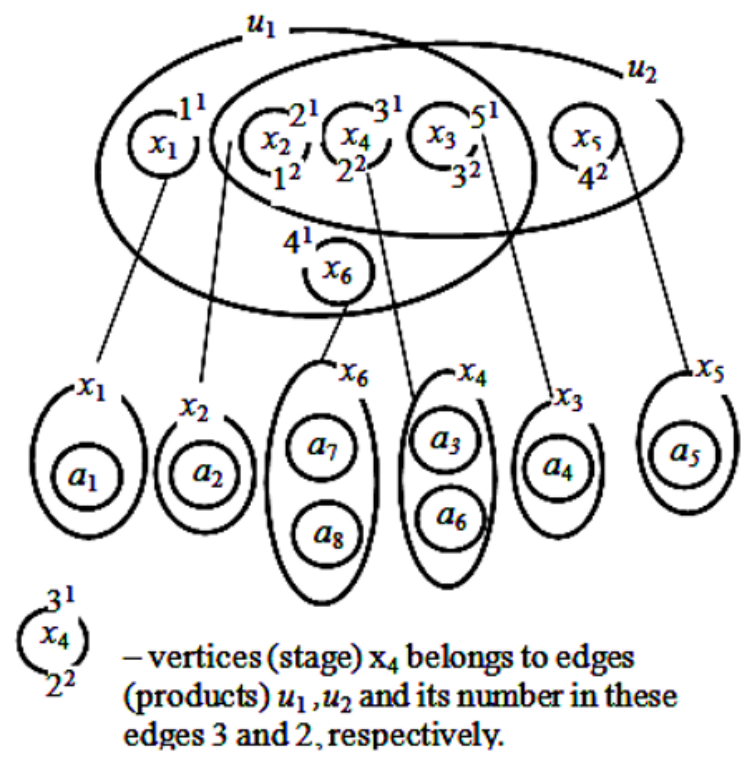

Fig. 3. Example of graphical representation of solution results for task of calculating the number and sizes of apparatuses

Figures 2 and 3 present an example of the initial hypergraph for solving the problem of calculating the number and sizes of apparatus and the solution variant. Oriented hypergraph $G_{g o}(X, U)$, as in the previous case, sets the sequence of stages for the output. Hypergraph $G_{g}(A, X)$ describes the apparatus of each stage.

As in the previous case the edges (products) $U$ have the following properties (colors): product name; volume of product; sizes of the finished product. Vertices (stages) $X$ - the 
name of stage; number of apparatuses, vertices (apparatuses) $A$ - type of apparatus; name of device; determining the geometric sizes (working volumes, surfaces) of the apparatus; start and end time for each batch of the product in given apparatus.

As you can see in Figure 3 as a result of the solution of the problem, a new stage $x_{6}$ is added, which includes two apparatuses $a_{7}$ and $a_{8}$ and a new vertex (apparatus) $a_{6}$ is added to the edge $x_{4}$.

As in finding the properties of vertices and edges of hypergraphs the solution of the problem in this case consists in changing the initial state of hypergraphs (adding vertices and edges) to reach the extremum of the criterion in accordance with the formulation of problem (1).

\section{Task of developing individual units of equipment}

As stated above, CTS consists of separate units (units of process equipment). Examples of individual units of equipment are capacitive apparatus, shell-and-tube heat exchanger, rectification column, etc.

Moreover, IM of a technical object represents it as a set of elements and connections between them and allows generating possible variants of its design that satisfy the initial data listed in the technical task $[7,8]$.

Knowledge representation in the form of products (rules) was chosen as a basic form of knowledge representation in IM. This is due to the fact that most of the requirements of regulatory documentation and the experience in the field of design are most simply, accurately and naturally formalized in the form of products. Other forms of knowledge representation in ILM are mathematical expressions or their systems (computational methods).

Formally, IM of a technical object will be represented in the form

$$
M=<E, M^{S}, M^{P}, M^{R}>
$$

where $E=\left\{e_{i},\right\}, i=\overline{1, N}$ - set of elements of technical object that is divided into the classes of functional elements $E^{b}=\left\{e_{k}\right\}, k \subset \overline{1, N}$ and connecting elements $E^{s}=\left\{e_{j}\right\}, j \subset \overline{1, N}$, each element is represented in the form $e=\langle P, Z n\rangle$, where $P=\{p\}-$ set of properties of this element, for example element type, structure (for complex elements), geometric and technical characteristics, production material, etc; $Z n=\{z n\}$ - set of possible values of properties of the given element, which can only take one value (for example, body material or diameter of cover), or several values at the same time (for example, for the coil, the "purpose" property can take the values "warm" and "cool").

$M^{S}=<E, O, Y^{e}, Y^{t}, Y^{k}, Y^{S}>-$ IM to determine the structure of TA, where $O_{-}$ AND-OR tree, elements of the developed apparatus, $Y^{e}$ - rules that determine the presence and number of functional elements of the apparatus; $Y^{k}$ - rules that determine the preliminary arrangement of elements relative to each other; $Y^{S}$-rules defining the types of connecting elements of a technical object.

$M^{P}=<E, Y^{b}, Y^{p p}, Y^{p e}>-$ IM to determine the parameters of TA, where $Y^{b}$ - rules and dependencies that determine the overall parameters of the apparatus as a whole; $Y^{p p}$ rules and dependencies that determine the values of single element parameters; $Y^{p e}-$ rules and dependencies that determine the values of the unitary parameters of the elements. 
$M^{R}=<E^{3 D}, T^{S P}, Y^{R}>-\mathrm{IM}$ of positioning $E^{3 D}=<O s, L, G r, S^{P}>-$ TA geometric 3D model, where $O s=\{o s\}-$ set of axes; $L=\{l\}-$ set of edges; $G r=\{g r\}-$ set of surfaces (edges); $S^{P}=\left\{s^{P}\right\}-$ set of interfaces (positioning links) between $O, L$ and $G r$; $T^{S P}$ - register of types of interfaces between base axes, edges and edges of elements; $Y^{R}$ rules that define the interfaces between the basic geometric parameters of the elements.

Individual units of equipment represent a system consisting of elements (shells, bottoms, supports, fittings, etc.) and the links between them.

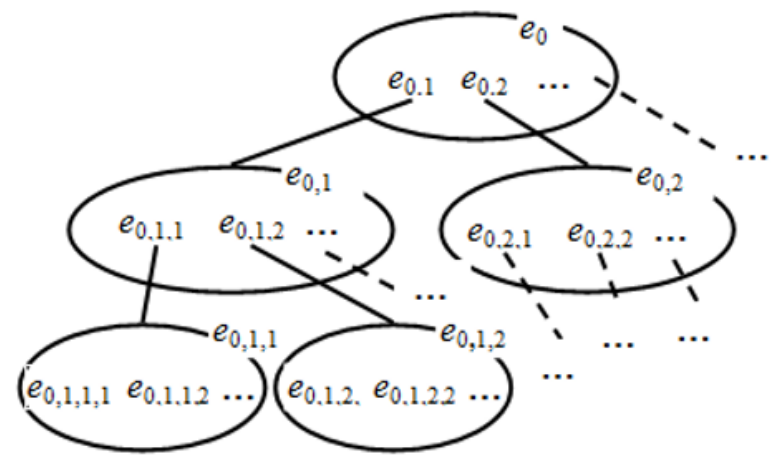

Fig. 4. Structure of process equipment.

Thus, the structure of a technical object can be represented as a hierarchy of hypergraphs (Figure 4). In this case, each element of the hierarchy has additional properties. For example, for a capacitive apparatus, the elements $e_{0,1}$ and $e_{0,2}$ will be a case and a drive. In turn, the elements of the case $e_{0,1,1}$ and $e_{0,1,2}-$ shell and bottom. In this case, the bottom will have a property of type, the value of which can be elliptical, conical or flat.

The main stages of designing technological equipment are:

- structural synthesis. Determination of the structure of the projected object based on IM for $M^{S}$ structure finding. The result is a list of the elements that make up the constructed object, and the links between the elements (the construction of hypergraph hierarchy, Figure 4);

- parametric synthesis. Determination of characteristics (properties) of elements, constructed object on the basis of IM for calculation of parameters of elements $M^{P}$;

At the base of structural synthesis there is AND-OR tree, which processing provides a designed object structure.

The processing of the tree can be performed using rules like "If ... then ..." [9-12]. For example: "If the arrangement of the capacitive apparatus "between floors", then the pillars are "legs";

Parts of "then" rule represent the vertices of the AND-OR tree. The "if" parts are the taxonomy (tree) of functions and operating conditions of equipment. Thus, the set of rules for selecting elements is an undirected ultragraph whose edges include the vertices of AND-OR tree (vertices receivers) and the tops of the function tree and equipment operating conditions (source vertices), figure 5 .

$O=(O V, O R)-$ AND-OR tree equipment, $O V=\left\{o v_{i}, i=0 . . I\right\}-$ set of equipment items $O R=\left\{o r_{k m}, k \in 0 . . I, m \in 0 . . I, k \neq m\right\}-$ links of class-subclass type, for example, "support leg support".

$U=(U V, U R)$ - taxonomy of functions and operating conditions of equipment, $U V=\left\{o v_{j}, j=0 . . J\right\}-$ set of functions and operating conditions of equipment, for example, 
the function - "mix", the operating conditions - "the environment in the apparatus is prone to precipitation", $U R=\left\{u r_{k m}, k \in 0 . . J, m \in 0 . . J, k \neq m,\right\}$ - links of the class-subclass type, for example "mixing-intensive mixing".

\section{AND-OR tree equipment, $O$}

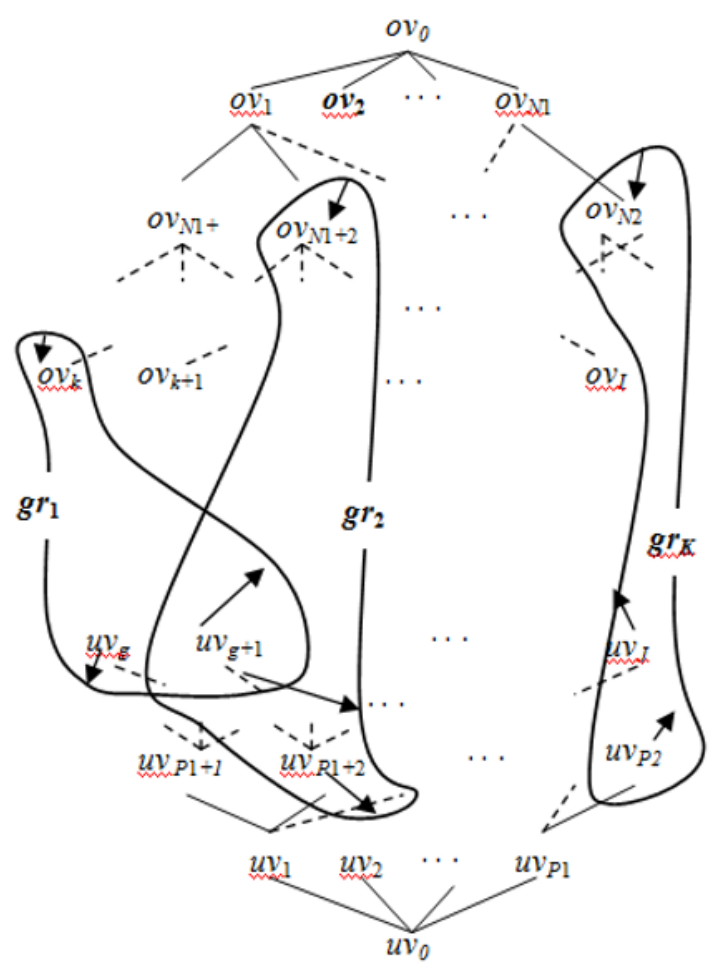

Taxonomy of functions and operating conditions of equipment, $U$

Fig. 5. Graphical representation of the structural synthesis of process equipment

$G=(G V, G R)-$ ultragraph of connections of vertices of AND-OR of tree of elements $O V=\left\{o v_{i}, i=0 . . I\right\}$ and tree vertices of function and operating conditions $U V=\left\{u v_{j}, j=0 . . J\right\} . G V=O V \cup U V-$ set of ultragraph vertices,

$G R=\left\{g r_{k}, k=1 . . K\right\}-$ set of ultragraph edges, $g r_{k}\left(Y_{k}\right)-k$ edge of hypergraph, $Y_{k}-$ set of vertices incident to $k$ edge, $Y_{k} \subset G V, Y_{k}=\left\{o v_{l}, U V 1\right\}, o v_{l} \in O V$ - vertices - equipment element, $U V 1 \subset U V$ - set of vertices from taxonomy of functions and operating conditions of equipment, $U V 1=\left\{u v_{c}, c \in J 1 \subset J\right\}$.

Thus, the edge of ultragraph, representing a rule (product) of the form "If ..., then", formally will be written as $\exists \bigcap_{c \in J l \subset J} u v_{c} \Rightarrow o v_{l}$.

\section{Results and discussion}

Information models are presented for solving the problems of calculating the determining sizes of CTS apparatuses and developing a separate technological apparatus. Different 
types of graphs are proposed for a formal description of the structure of developed CTS and technological devices.

The presented results are used in the development of information support for decisionmaking in the design of MCI.

This work was financially supported by Ministry of Education and Science of the Russian Federation within the basic part (project 8.7082.2017/8.9)

\section{References}

1. 1. A.N. Budyakov, K.G. Getmanova, M.G. Matveyev. Vestnik of VSU: series "System Analysis and Information Technology", 2, 66-71 (2017).

2. 2. B. Pozdneev, F. Busina, A. Ivannikov. Smart Innovation Systems and Technologies, 59, 73-83 (2016).

3. 3. B. Pozdneev, F. Busina, M. Sutyagin, A. Levchenko, V. Tikhomirova. EDULEARN 16 Proceedings, 2118-2125 (2016).

4. 4. A. Ivannikov, V. Kulagin, A. Romanov, B. Pozdneev. EWDTS-2016 Proceedings, DOI: $10.1109 /$ EWDTS.2016.7807712.

5. 5. A.B. Borisenko, S.V. Karpushkin. Journal of Computer and Systems Sciences International, 53, 5, 410-419 (2014).

6. 6. E.N. Malygin, S.V. Karpushkin, M.N. Krasnyanskiy, A.V. Ostroukh. AmericanEurasian Journal of Agricultural \& Environmental Sciences, 3, 447-453 (2015).

7. 7. V.G. Mokrozub, V.A. Nemtinov. A.V.Mokrozub. Chemical and Petroleum Engineering, 53, 5-6, 326-331 (2017).

8. 8. V.G. Mokrozub, V.A. Nemtinov Chemical and Petroleum Engineering, 51, 7, 487492 (2015).

9. 9. A.A. Bolshakov. Intellektualnye-sistemy-upravleniya-organizacionnotekhnicheskimi-sistemami [Intellectual systems of management of organizational and technical systems] Moscow: Hot line-Telecom, 2016. (in Russian)

10. 10 T.B. Chistyakova, O.G. Boykova, N.A. Chistyakov. Intellektualnoe-upravleniemnogoassortimentnym-koksohimicheskim-proizvodstvom [Intellectual management of multi-assortment coke-chemical production] St. Petersburg: Educational Center «Profession», 2010. (in Russian)

11. 11. E.N. Malygin, V.G. Mokrozub, V.A. Nemtinov. MATEC Web of Conferences. 129, 01009 (2017).

12. 12. A.F. Egorov, T.V. Savitskaya, V.A. Kolesnikov, Yu. A. Kuzmina. Tsvetnye Metally [Non-ferrous metals], 4, 78-84 (2015). 